Epidemiology

\title{
Cheerleader injuries: a Brazilian cross-sectional study
}

\author{
Isabela Bianchini Marolde ${ }^{1,2} *$ (D), Cristiano Carvalho ${ }^{1,2}$ (D), \\ Paula Regina Mendes da Silva Serrão ${ }^{1,2}$ (D) \\ ${ }^{1}$ Universidade Federal de São Carlos, Departamento de Fisioterapia, São Carlos, SP, Brasil; \\ ${ }^{2}$ Universidade Federal de São Carlos, Programa de Pós-Graduação em Fisioterapia, São Carlos, \\ SP, Brasil.
}

Associate Editor: Romulo A. Fernandes, Universidade Estadual Paulista (UNESP), Presidente Prudente, SP, Brasil.

\begin{abstract}
Aim: This study aimed to characterize the types, frequency, body locations, and activities related to cheerleading injuries. Methods: This was an Internet-based survey among Brazilian cheerleaders at the competitive national level. Data collected from participants included information about previous injuries (number, location, anatomical structure), age, sex, height, weight, occupation, cheerleading position, number of years of experience, and frequency and hours of training per week. The significance level was set at $\alpha=0.05$. Results: In total, 113 cheerleaders answered the online questionnaire, and 230 injuries were reported. The most affected anatomical site was the wrist with $14.8 \%$ of the injuries, followed by the ankle $(11.3 \%)$, and the hand and fingers $(9.1 \%)$. There is a direct association between the occurrence of injuries and experience cheerleading practice $(p=0.002)$. Conclusions: There is a considerable number of injuries in cheerleading, mostly occurring in the upper limbs. Specific preventative education or training could be directed at these athletes, with an emphasis on reducing upper limb injuries.
\end{abstract}

Keywords: athletes, epidemiology, injuries, wrist, sports.

\section{Introduction}

Cheerleading is a physical activity that originated at the University of Minnesota around the year $1898^{1,2}$. Cheerleading became more popular over the years, and from 1990 to 2003 the number of cheerleaders in the world increased from 600 thousand to 3.6 million $^{3-6}$. The sport includes dance moves, tumbling, complex gymnastics maneuvers, stunts, and pyramids held in groups performed during a piece of music to form what is called a cheerleading routine. Depending on the intensity and frequency of these complex movements, injuries can occur.

There are three main cheerleader positions: base, flyer, and spotter ${ }^{4,7}$. Together, they aim to form the stunt position (where an athlete is lifted by one or more athletes). The base is the cheerleader that provides primary support for the flyer. Their functions are to hold, lift and/or toss the flyer in the air. The flyer is the cheerleader that is lifted and supported by the base in the stunt, performing several maneuvers in this position ${ }^{8}$. The spotter is a cheerleader whose main responsibility is to protect the flyer from possible falls during the stunt formation ${ }^{4,7}$. Another function performed individually by the cheerleaders is the tumbling maneuvers, that is, acrobatic skills performed along the surface ${ }^{4}$ and which are similar to solo routines presented by artistic gymnasts.
Although it is now a widespread sport, cheerleading in Brazil is very recent and the sport is still gaining visibility and recognition. The Brazilian Union of Cheerleading was founded in 2008, and Brazil joined the International Cheer Union (ICU), a governing body of the sport in the United States, in the same year ${ }^{9}$. Since 2011, Brazil has been promoting national cheerleading competitions, which involve the participation of elementary school, high school, university, and All-Star teams.

As cheerleading evolved to a competitive level, the difficulty of maneuvers increased, consisting of gymnastics-style tumbling, fast-paced floor routines with leaps and jumps, and complex stunts such as forming human pyramids and tossing athletes in the air. This evolution in athletic demands and the increase in the number of participants are the primary reasons for the increase in the number of injuries ${ }^{10}$. According to an epidemiological study ${ }^{11}$ conducted in the United States, most injuries in cheerleaders occur in the lower limbs (42\%), with the most affected regions being the ankles and knees; followed by injuries to the trunk $(26 \%)$, with a higher prevalence in the lower back and shoulders; the upper limbs (21\%), with a predominance of injuries in the wrist and hand region; and finally the neck (11\%). Other studies have shown that the most common injuries are strains and sprains, abrasions, contusions, bruises, concussions, and dislocations ${ }^{8}$ result- 
ing from the movements of the flyer during stunt dismounts, failure to complete a maneuver, lifting the flyer, tossing the flyer in the air, or when a fall occurs ${ }^{12}$.

Despite the lack of studies on Brazilian cheerleaders, if we consider modalities that present a similar movement pattern, we can indicate the national panorama. Brazilian studies have already established a high frequency of injuries in dancers and gymnasts ${ }^{13-15}$. While athletes in artistic gymnastics had a high prevalence of injuries in the upper limbs (especially the wrist and shoulder), ballet dancers, ballroom dancers, and trampoline gymnasts had more injuries in the lower limbs. Ankle sprains stand out with an occurrence of $25.9 \%$ to $67.6 \%$ having been reported ${ }^{13-15}$. One of the differences that can be highlighted between these modalities is that in artistic gymnastics there is a high frequency of weight-bearing in the upper limbs. This is a possible explanation for the pattern of injuries in the upper limbs ${ }^{13}$.

There are no epidemiologic studies on Brazilian cheerleaders' injuries, despite cheerleading becoming a widespread sport in the university environment. This type of information is a prerequisite to guide future studies on disciplines, treatments, and prevention in a given population. Injuries can negatively impact the quality of life of athletes, resulting in withdrawal from sports participation, and depending on the degree of the injury it could also cause functional damage in the future. The first step in injury prevention is the identification of which injuries are most common in a particular sport, thus, the objective of this study was to characterize the main injuries in cheerleaders, considering types, frequency, locations, and mechanism of injuries, and the factors associated with these injuries, using an Internet-based survey. We hypothesize that injuries are common in cheerleading sports in Brazilian athletes and that the upper limbs should be more affected due to the constant use of this segment for weight-bearing during maneuvers.

\section{Methods}

\section{Study design}

This was an Internet-based survey, conducted consistent with the methods in the CHERRIES Checklist ${ }^{16}$ and the STROBE Statement ${ }^{17}$. The study was approved by the Human Research Ethics Committee of Universidade Federal de São Carlos (UFSCar) (CAAE: 60262316.9.0000.5504).

\section{Development and pre-testing}

Data collection was performed through a self-completion questionnaire. The questions were developed by the three authors based on prior studies and recommendations ${ }^{11,12}$. A first version of the questionnaire was assessed by two experts and the resulting suggestions were discussed between authors. All authors were in complete agreement with the final version of the questionnaire. The following step was to conduct a pilot test before releasing the questionnaire: 30 people, including cheerleading athletes, non-athletes, and health professionals linked to the Physical Therapy Department at the UFSCar completed the questionnaire to verify comprehension and adapt the vocabulary of the questions, as necessary with the goal to create the final version of the questionnaire.

\section{Recruitment process and description of the sample}

An open survey was used to collect data from participants. Brazilian cheerleaders who were older than 15 years old were included in this study. A list of the teams to be contacted was compiled, starting with the teams that participated in the Cheer and Dance National (Brazil) Championship in 2016, then adding the teams listed on the website of the Brazilian Cheerleading Union and the teams found on social networks. The initial contact with potential participants was made on the Internet, and the survey was announced to cheerleading coaches by email and to cheerleading teams by social media. We used a convenience sample due to the impossibility of calculating a representative sample, as no list involving all cheerleading teams in Brazil, or the number of participants, was found. Individuals who participated in teams with no competitive purpose were not included in this study, nor were individuals who had sustained injuries not related to the practice of cheerleading.

\section{Survey administration}

The questionnaire was developed on an online platform and its link was sent to the national cheerleading teams via an inbox message of a social network and via email, along with detailed information about the study and the participant consent form. Two weeks after the questionnaire was sent, a reminder message was sent in the same manner as the first contact. The completed questionnaires were automatically sent to an automatic database to capture the information. Only those who had the questionnaire link could access the online survey. The participants did not receive monetary incentives for their participation. Nevertheless, we made it clear in the information sent to participants that the results of the current study would help us understand the burden of injuries in cheerleaders and may guide the development of preventative strategies.

The survey link was available online from the June $30^{\text {th }}$ until August $7^{\text {th }}, 2017$. The online questionnaire consisted of four pages and each page had an average of 13 questions. The branching logic feature was used with some questions, meaning that additional questions would be provided based on the answer to a previous question. The survey questionnaire (Supplemental material) was composed of 51 questions divided into three sections: 
demographic (sex, age, height, body mass, body mass index [BMI], and occupation), sports (cheerleading position, years of experience in cheerleading, training frequency, training duration), and injury characteristics (number of injuries, affected site, affected anatomical structure, activity related to the injury). There were explanations throughout the questionnaire, in the questions, section headings, and in the consent form so that the athletes could answer the questions in the best possible way. The injury was defined in the questionnaire as any physical complaint made by an athlete resulting from cheerleading competition or training, regardless of the need for medical evaluation or withdrawal from cheerleading activities. This definition was designed to facilitate the participants' understanding and is in accordance with the definition proposed by the International Olympic Committee Consensus Statement ${ }^{18}$. The participants were asked to report any injuries suffered during the practice of cheerleading since the beginning of their contact with the sport. It was also asked the cheerleaders to consider the main position in which they practice (that is, the one to which they devoted more time) and to report the current frequency and time of practice.

A completeness check was automatically performed for each section of the questionnaire to ensure that all questions had been answered by the participant before being allowed to complete the next survey section. If needed, respondents were able to review and change their answers using the "Back" button feature. The survey system could not identify the IP address of the participant's computer to verify possible duplicate entries. However, the username used by the participant was stored together with the search results, allowing the identification of duplicate entries by the same user. In the case of doubleentry, the first entry would have been kept for analysis, but no duplicate username was observed in this study. No time limit was used to allow the completion of the questionnaire.

\section{Statistical analysis}

Only data from participants who fully completed the survey questionnaire were analyzed. Statistical analysis was performed using IBM SPSS Statistics software (IBM SPSS Statistics for Windows, Version 19.0. Armonk, NY: IBM Corp.), and it was carried out in 2018-2019. Quantitative data were expressed as mean \pm standard deviation and qualitative data as simple frequency. The prevalence of injuries was calculated by dividing the number of injured athletes by the total number of athletes. Associations between the occurrence of injury (whether the athlete had or not sustained an injury) and the clinical (sex, age, BMI) and training variables (cheerleading position - flyer and base -, training duration, years of experience in cheerleading, carrying out another physical activity other than cheerleading and sports practice before cheerleading) related independent variables were tested using the ChiSquare test. Association between the occurrence of injury and training frequency was tested using Fisher's exact test. All types of bases were grouped in the analysis because they have a common function during the stunt formation of first supporting and then catching the flyer during stunt dismounts. The variables that showed significant association (occurrence of injuries and experience cheerleading practice) were analyzed using logistic regression (Hosmer and Lemeshow correction was applied), which generated odds ratios (OR) and $95 \%$ confidence intervals $(\mathrm{CI})$. The significance level was $\alpha=0.05$.

\section{Results}

A total of one hundred fourteen cheerleaders completed the online questionnaire. One participant was excluded from the analysis for being younger than 15 years old. Thus, our data sample contained 113 cheerleaders. The characteristics of the participants are described in Table 1 . Most of the athletes $(61.9 \%, \mathrm{n}=70)$ reported having more than one year of cheerleading experience. The most common training frequency was

Table 1 - Descriptive data for cheerleading participation.

\begin{tabular}{lc}
\hline Characteristics & Athletes $(\mathbf{n}=\mathbf{1 1 3}) \mathbf{n}(\mathbf{\%})$ \\
\hline Age $($ years $)$ & $21.0 \pm 2.4$ \\
BMI $\left(\mathrm{kg} / \mathrm{m}^{2}\right)$ & $21.9 \pm 3.0$ \\
Sex & \\
Female & $85(75.2)$ \\
Male & $28(24.8)$ \\
Position & \\
Base & $71(62.8)$ \\
$\quad$ Back spot & $19(16.8)$ \\
$\quad$ Front spot & $4(3.5)$ \\
$\quad$ Side base & $48(42.5)$ \\
Flyer & $40(35.4)$ \\
Spotter & $2(1.8)$ \\
Year of experience & \\
Less than 6 months & $22(19.5)$ \\
6 months - 1 year & $21(18.6)$ \\
More than 1 year & $70(61.9)$ \\
Training frequency (days/week) & \\
1-2 & $41(36.3)$ \\
3-4 & $63(55.8)$ \\
5-7 & $9(7.9)$ \\
Training duration (hours/session) & \\
Bess than 1 & $2(1.8)$ \\
More than 2 & $61(54.0)$ \\
\hline . & $50(44.2)$ \\
\hline
\end{tabular}

Legend: BMI: body mass index. 
three to four times a week $(55.8 \%, \mathrm{n}=63)$ with a training duration between one and two hours per session $(54.0 \%$, $\mathrm{n}=61)($ Table 1).

There were 230 injuries reported. More than half of the athletes $(55 \%, \mathrm{n}=62)$ reported sustaining at least one previous injury during cheerleading practice and $4 \%$ $(\mathrm{n}=5)$ reported also sustaining at least one previous injury during a cheerleading competition (no athlete reported injury only during competition). The mean \pm standard deviation number of injuries per athlete was $2.04 \pm 2.92$ when considering all 113 participants or $3.71 \pm 3.06$ injuries per previously injured athletes. Among injuries reported by cheerleaders, the wrist was the most often injured site with 34 injuries (14.8\%), followed by the ankle with 26 injuries $(11.3 \%)$, and then the hand and the fingers with 21 injuries (9.1\%) (Figure 1). The most injured anatomical structure was muscles $(20.5 \%)$ and the stunt was the most common cheerleading activity related to injuries was, with $31 \%$ of the injuries occurring during its formation and $25.4 \%$ during its dismount (Table 2).

When asked about what they believed had caused their injuries, most of the athletes reported believing that their injuries were caused by misuse of cheerleading technical gestures $(24.8 \%)$, followed by performing a sudden gesture/movement $(20.4 \%)$ and physical tiredness and/or general fatigue (18.6\%) (the athletes could choose more than one answer to this question, so the same athlete can be represented more than once in the percentages). Furthermore, $14,2 \%$ believed their injuries were caused by continuous repetition of the same movements (overuse), $9.7 \%$ due to an inadequate recovery from previous injuries
Table 2 - Injury characteristics.

\begin{tabular}{lc}
\hline Characteristics & Number of injuries (\%) \\
\hline Injured structure & $12(9.1)$ \\
Bone & $1(0.8)$ \\
Bursa & $6(4.5)$ \\
Cartilage & $23(17.4)$ \\
Joints & $19(14.4)$ \\
Ligaments & $27(20.5)$ \\
Muscle & $2(1.5)$ \\
Nerve & $9(6.8)$ \\
Skin & $15(11.4)$ \\
Tendon & $18(13.6)$ \\
Did not know & \\
Action leading to Injury & $5(7.0)$ \\
Aerial figures & $8(11.3)$ \\
Jumps & $18(25.4)$ \\
Stunt dismount & $22(31.0)$ \\
Stunt formation & $8(11.3)$ \\
Tumbling & $10(14.1)$ \\
Others &
\end{tabular}

Others: warm-up run, run for routine preparation, basket (that is an advanced type of stunt in which the bases toss the flyer in the air and has several possible variations), stretching, pyramid formation.

and $6.2 \%$ of the athletes believed that the type of floor they trained on was the cause of the injury.

The occurrence of injuries was associated with years of experience $(p=0.002)$ (Table 3$)$. For athletes with more than 1 year of experience, the prevalence of sustaining a

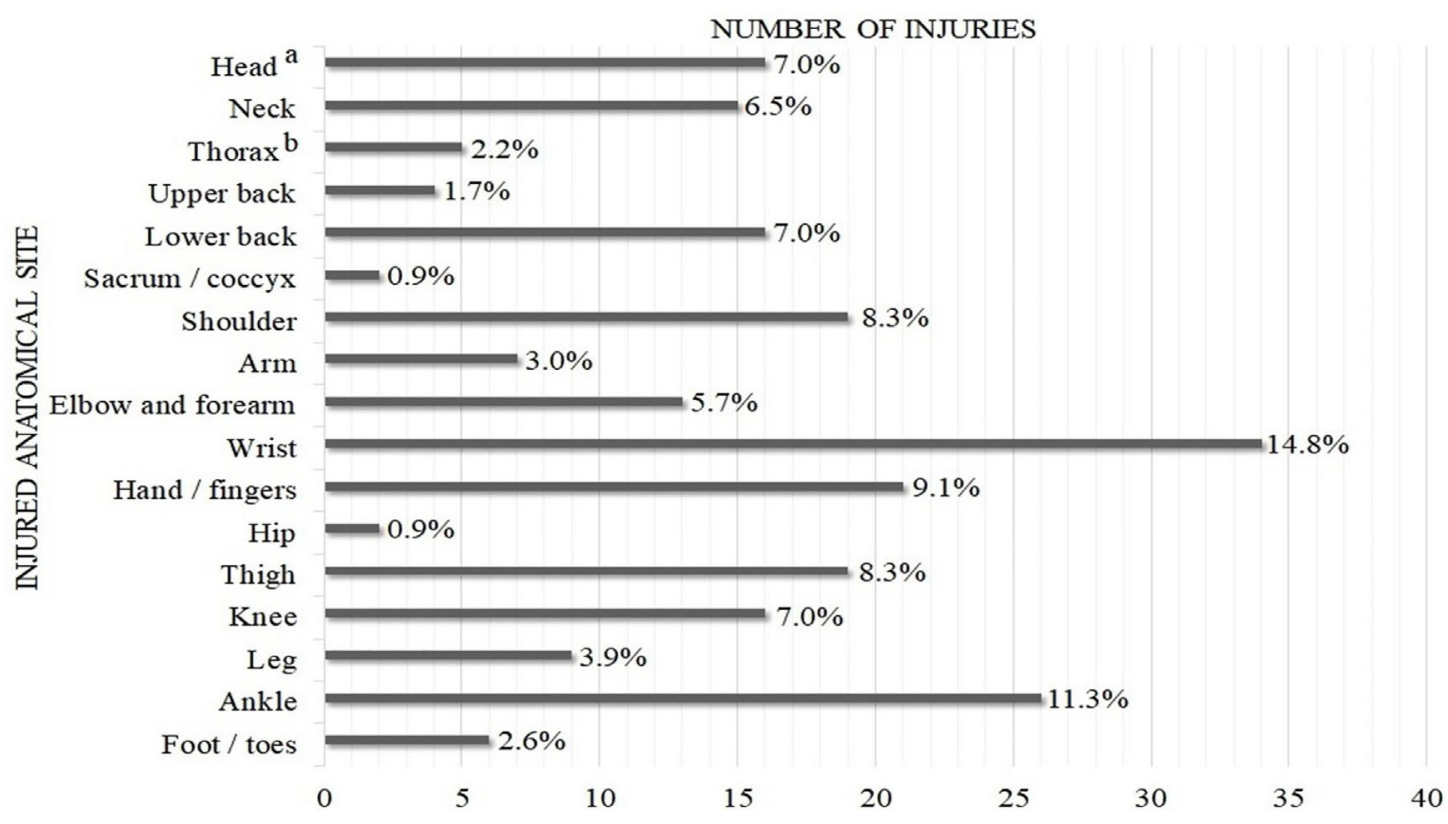

Figure 1 - Percentage and number of injuries per anatomical site. ${ }^{\mathrm{a}} \mathrm{Head}$ : includes eyes, ears, mouth, and nose. ${ }^{\mathrm{b}}$ Thorax: includes rib and sternum, abdomen. In total, 230 injuries were reported. 
Table 3 - Occurrence of injuries according to descriptive and cheerleading characteristics

\begin{tabular}{|c|c|c|c|}
\hline \multirow[t]{2}{*}{ Variables } & \multicolumn{3}{|c|}{ Occurrence of injuries } \\
\hline & $n(\%)^{d}$ & Chi-square (p-value) & Logistic Regression OR $(95 \% \mathrm{CI})$ \\
\hline Sex & & 0.3 & \\
\hline Female $(\mathrm{n}=85)$ & $49(79)$ & & - \\
\hline Male $(\mathrm{n}=28)$ & $13(21)$ & & - \\
\hline Age (years) & & 0.07 & \\
\hline $15-19(\mathrm{n}=38)$ & $26(41.9)$ & & - \\
\hline $20-24(\mathrm{n}=61)$ & $31(50)$ & & - \\
\hline $25-29(n=14)$ & $5(8.1)$ & & - \\
\hline BMI & & 0.9 & \\
\hline Underweight $(\mathrm{n}=10)$ & $5(8.1)$ & & - \\
\hline Normal $(n=89)$ & $49(79)$ & & - \\
\hline Overweight $(\mathrm{n}=14)$ & $8(12.9)$ & & - \\
\hline Position & & 0.2 & \\
\hline Flyer $(n=40)$ & $25(62.5)$ & & - \\
\hline $\operatorname{Base}(\mathrm{n}=71)^{\mathrm{e}}$ & $35(49.3)$ & & - \\
\hline Training frequency (days/week) & & 0.7 & \\
\hline $1-2(n=41)$ & $21(51.2)$ & & - \\
\hline $3-4(n=63)$ & $37(58.7)$ & & - \\
\hline $5-7(n=9)$ & $4(44.4)$ & & - \\
\hline Training Duration (hour/session) & & 0.8 & \\
\hline Less than or up to $2(n=63)$ & $34(54.8)$ & & - \\
\hline More than $2(n=50)$ & $28(45.2)$ & & - \\
\hline Amount of experience & & 0.002 & \\
\hline Less than 6 months $(\mathrm{n}=22)$ & $5(8.1)$ & & 1.0 \\
\hline 6 months -1 year $(n=21)$ & $11(17.7)$ & & $3.7(1.0,13.9)$ \\
\hline More than 1 year $(n=70)$ & $46(74.2)$ & & $6.5(2.1,19.8)$ \\
\hline Carrying out a physical activity other than cheerleading & & 0.2 & \\
\hline Yes $(n=49)$ & $30(48.4)$ & & - \\
\hline No $(n=64)$ & $32(51.6)$ & & - \\
\hline Sports practice before cheerleading & & 0.2 & \\
\hline Yes $(n=82)$ & $48(77.4)$ & & - \\
\hline No $(n=31)$ & $14(22.6)$ & & - \\
\hline
\end{tabular}

Notes: Hosmer-Lemeshow test $=1.0 ; \mathrm{CI}=$ confidence interval; $\mathrm{OR}=$ odds ratio.

${ }^{\mathrm{d}}$ The data are the number (\%) of participants who sustained an injury within that subgroup.

For the analysis of Chi-square and logistic regression the all the athletes in the Base position were grouped (back spot, front spot and side base). Furthermore, the position of spotter was not included in the analysis due to the small representation in our sample $(\mathrm{n}=2)$ and the fact that they both sustained injuries.

Boldface indicates statistical significance $(\mathrm{p} \leq 0.05)$.

previous injury was $74.2 \%$, compared to $25.5 \%$ for those with less than 6 months of experience (Table 3). Cheerleaders with experience practice between 6 months and 1 year were 3.7 times more likely to report injuries when compared to athletes with less than 6 months of experience (Table 3). And cheerleaders with more than one year of experience practice were 6.5 times more likely to report injuries when compared to athletes with less than 6 months of experience. The descriptive characteristics of the sample (sex, age, BMI, carrying out another physical activity other than cheerleading, and sports practice before cheerleading) and other cheerleading variables (cheerleading position, training duration, and frequency) did not show a significant association with the occurrence of injuries $(\mathrm{p}>0.05)$.

\section{Discussion}

This is the first study to describe the prevalence of injuries in Brazilian cheerleaders. In addition, our findings 
showed relevant data about the practice of cheerleading in Brazil. We found that the wrist was the most common injured site among the athletes, accounting for $14.8 \%$ of the total injuries. The ankle and the hand or fingers were the next most affected sites, representing $11.3 \%$ and $9.1 \%$ of all injuries, respectively. The most injured anatomic structure was the muscle tissue $(20.5 \%)$. Most injuries occurred during practice $(97 \%)$ when compared to competitive events (3\%) and the moment of injury most common was during attempting a stunt $(56.4 \%)$; we investigated this moment of injury dividing the stunt into two moments: stunt formation, in which $31 \%$ of all the injuries occurred; and dismount, with $25.4 \%$ of all injuries. Our findings also revealed that the greater the cheerleading experience the greater the occurrence of injuries.

Based on the available literature and the specific characteristics of the functions performed by cheerleading athletes, we expected to find a greater prevalence of ankle and wrist injuries ${ }^{1,11,12,19}$. Previous studies have shown that the ankle is the anatomical site most affected by injuries in cheerleaders with a prevalence of $12-44.9 \%$ of the injuries ${ }^{11,12,19}$ while the wrist was the second most affected site $(19.3 \%)^{19}$ and a very common site of reinjury $(15 \%-18 \%)^{11,12}$. In general, studies have demonstrated that the lower limb was the most injured segment or had one of its joints as the most affected ${ }^{2,4,11,12,19}$. However, our collected data showed a higher prevalence of upper limb injury (41\%), with the wrist being the most injured anatomical site of the body (14.8\%).

Despite contradicting the international literature, these results are consistent if we consider that teams with more stunts and catches are more likely to injure the upper body $^{20}$ than teams in which predominate tumbling maneuvers. Thus, the ankle injuries reported in previous studies may be related to the greater number of tumbling skills in relation to Brazilian teams. Comparing this data with the Brazilian literature, we can relate it to athletes of artistic gymnastics who present high frequencies of injury in upper limbs, due to the characteristics of weight-bearing in their modality ${ }^{13}$. In addition, the major percentage of the athletes who participated in the research had stunts as their main function in the team. This is strictly related to the high incidence of wrist injuries.

Furthermore, these findings are logical in view of the big stress to which this joint is exposed: high and repetitive impact, axial load and rotational forces strike the wrists of these athletes ${ }^{21}$ during tumbling and stunts. Moreover, wrist injuries can mainly be attributed to the cheerleaders whose main function on the team is the base, and among the base types, the main ones to have this joint affected are the side bases (in our study, $65 \%$ of the 34 wrist injuries occurred in side bases and the other 35\% occurred in back spots, flyers, and spotters); also, the side base was the most common position among the consulted athletes. The bases are responsible for supporting the fly- ers, as well as raising, tossing, and securing them. The wrist base remains hyperextended most of the time during the execution of these tasks. In this way, the joint undergoes repetitive stress over an extreme range, and this may be one of the factors that lead these athletes to the higher prevalence of these injuries.

We found that the ankle is the second most affected site of injuries $(11.3 \%)$. The literature suggests that the main causes of ankle injuries are constant landings on hard surfaces $^{1}$ and falls ${ }^{1,12}$. Most athletes reported performing their training on suitable surfaces such as mats or platforms $(67.3 \%)$, however, $17.7 \%$ reported training on the grass and $11.5 \%$ on a hard surface. As only $6.2 \%$ of the athletes believed that the type of floor they trained on was the cause of the injury, this reinforces that most athletes trained on an adequate surface and collaborates with the information that ankle injuries were more common in flyers $(46.1 \%$ of the ankle injuries occurred in flyers and the other $53.9 \%$ occurred in bases and spotters), since its main function is to perform acrobatics while being lifted and standing over the hands of the bases.

Our analysis showed an association between the occurrence of injuries and the years of experience in cheerleading practice, appointing those athletes who practice for more than 1 year are 6.5 times more likely to report injuries than those with less than 6 months of experience. These results coincide with findings of study ${ }^{12}$ that reported that most of the injured cheerleaders were the more experienced ones. This could be explained by an accumulation of injuries that occurred during the practice of the sport. Moreover, there is also the possibility that more experienced athletes perform more complex maneuvers being exposed to increased risks ${ }^{20}$. For a better understanding of this matter, it is necessary to conduct a study comparing the injuries of athletes with different levels of experience during a shorter and more recent period (in the last year, for example).

No associations were found between the occurrence of injuries and sex, age, BMI, carrying out another physical activity other than cheerleading and sports practice before cheerleading. An epidemiological study of sports injuries in Canadian children and adolescents reported that men are injured more frequently during sports than women $^{22}$. Seventy-one percent of sports injuries were reported by men, specifically in 11 out of the 13 sports analyzed $^{22}$. In contrast, Lin et al. ${ }^{23}$ showed that the prevalence of sports injuries between the sexes varies according to the type of injury, for example, bone stress injuries and concussions are more common in female athletes while the rate of anterior cruciate ligament injuries among the sexes is similar in professional sports. However, Gornitzky et al. ${ }^{24}$ showed that female athletes have a substantially higher risk of injury to the anterior cruciate ligament than male athletes. Thus, there is no consensus in the literature on which sex has the highest rate of occur- 
rence of sports injuries. It is known that the etiology of sports injuries is multifactorial, covering a variety of factors ${ }^{25,26}$. Further studies are needed to investigate the association of occurrence of injuries with sex, BMI, and physical fitness attributes include the components of agility, balance, body composition, coordination, flexibility, muscular endurance, and muscular strength.

With our results, coaches and clinicians should be aware that wrist and ankle injuries are the most common in this population and use this information to develop specific preventive training. The data we collect does not provide any information about the composition of the cheerleading's technical team, but analyzing the Brazilian context and considering that this is still an emergent sport and that most teams are from universities, we believe that athletes do not have immediate access to a multidisciplinary team. This lack of support in the technical teams can also be related to the high occurrence of injuries, as already verified in a previous study ${ }^{27}$ that found a higher risk of injuries in cheerleaders supervised by a coach with low qualifications. Even so, our study brings important data about specific characteristics of cheerleading in the Brazilian population that can be helpful to coaches who are willing to learn more about the epidemiology of the sport to improve their training and also for clinicians to know important factors related to the sport and the specifics of their injuries.

As the limitation of this study, we did not investigate the time lost from the sport in each injury and neither the impact that these injuries had on the athlete's quality of life. Besides, the lack of information on the competitive level of the athletes prevented a deeper analysis of their association with the prevalence of injuries. In our survey, we collected data for injuries sustained in cheerleadingrelated practice since the beginning of the contact with the sport, without determining a shorter and more recent period that would allow us to compare the injuries between athletes with different levels of experience in the same period. So, for future studies, we strongly suggest that a period be determined for the collection of injuries suffered by cheerleaders. An inherent limitation of the design of our study is the impossibility of calculating the questionnaire response rate: because it was an online questionnaire released through the teams' e-mail addresses and social networks, it was not possible to identify how many people received the questionnaire. The reliability of the questionnaire was not tested. However, it is emphasized that the questions were developed by the three authors based on prior studies and recommendations ${ }^{11,12}$. Two experts checked its content and some volunteers tested its intelligibility, we considered that the questionnaire provided adequate answers. Future studies could be designed to test its reliability and also evaluate the competitive level of the athletes to allow a deeper analysis of their association with the prevalence of injuries.

\section{Conclusions}

This study is the first to describe the prevalence of injuries in Brazilian cheerleaders, presenting a list of the main injuries and their characteristics in athletes in an emerging sport in Brazil. In summary, there is a high prevalence of cheerleader injuries in Brazil and these injuries mostly occurred in the upper limbs, with wrist injuries being the most commonly affected site, followed by ankle and hand or finger injuries. Furthermore, the greater experience in cheerleading practice is associated with a greater occurrence of injuries in these athletes. Coaches and health professionals involved with the monitoring or care of cheerleaders should be aware that athletes from different cheerleading positions have different injury prevalence, so this is a factor that must be considered when developing cheerleading training. Taking into account that many of the Brazilian cheerleading teams do not have access to a multidisciplinary team, our data can be used to support these athletes, serving as a basis for the creation of preventive training. Thus, it should be taken into consideration a large number of injuries present in cheerleading and considering that they are mainly manifested in the upper limbs when aiming to develop specific training for injury prevention. In addition, based on our results, health professionals can select tests and evaluation procedures to screen athletes for injuries.

\section{Acknowledgments}

The authors would like to thank the support of União Brasileira de Cheerleading (UBC) and their assistance in disseminating this research and the involvement of the participants in this study. The authors are also grateful to Ana Paula Rodrigues Rocha and Tatiana Oliveira Sato for their assistance in reviewing the statistical analysis. This study was supported by the Programa Institucional de Bolsas de Iniciação Cientifica (PIBIC) / Conselho Nacional de Desenvolvimento Científico e Tecnológico (CNPq, Process number 103101/2017-9), Brazil. I.B.M. was $\mathrm{PIBIC} / \mathrm{CNPq}$ fellowship.

\section{References}

1. Waters N. What goes up must come down! A primary care approach to preventing injuries amongst highflying cheerleaders. J Am Acad Nurse Pract. 2013;25(2):55-64.

2. Shields BJ, Smith GA. Cheerleading-related injuries to children 5 to 18 years of age: United States, 1990-2002. Pediatrics. 2006;117(1):122-9.

3. Hardy I, McFaull S, Saint-Vil D. Neck and spine injuries in Canadian cheerleaders: An increasing trend. J Pediatr Surg. 2015;50(5):790-2.

4. Council on Sports Medicine and Fitness. Cheerleading Injuries: Epidemiology and Recommendations for Prevention. Pediatrics. 2012;130(5):966-71. 
5. History of cheerleading. International Cheer Union. http:// cheerunion.org/history/cheerleading/. Accessed October 19, 2016.

6. Laudner KG, Metz B, Thomas DQ. Anterior glenohumeral laxity and stiffness after a shoulder-strengthening program in collegiate cheerleaders. J Athl Train. 2013;48(1):25-30.

7. Waters N. What goes up must come down! A primary care approach to preventing injuries amongst highflying cheerleaders. J Am Acad Nurse Pract. 2013;25(2):55-64. doi:10.1111/1745-7599.12000

8. Carrier J, McKay D. Complete Cheerleading. Champaign, IL: Human Kinetics; 2006.

9. Cheerleading. União Brasileira de Cheerleading. http:// www.cheerleading.com.br/ubc/cheerleading.php. Accessed March 8, 2016.

10. Naiyer N, Chounthirath T, Smith GA. Pediatric Cheerleading Injuries Treated in Emergency Departments in the United States. Clin Pediatr (Phila). 2017;56(11):985-92.

11. Shields BJ, Smith GA. Epidemiology of strain/sprain injuries among cheerleaders in the United States. Am J Emerg Med. 2011;29(9):1003-12.

12. Shields BJ, Smith GA. Epidemiology of Cheerleading Stunt-Related Injuries in the United States. J Athl Train (National Athl Trainers' Assoc. 2009;44(6):586-94.

13. Bolling C, Leite M. Difference in injury profile in Trampoline and Artistic Gymnastics. J Sci Med Sport. 2012;15 (Supplement 1):S127-8. doi:10.1016/j.jsams.2012.11.307

14. Costa MSS, Ferreira AS, Orsini M, Silva EB, Felicio LR. Characteristics and prevalence of musculoskeletal injury in professional and non-professional ballet dancers. Brazilian J Phys Ther. 2016;20(2):166-75. doi:10.1590/bjptrbf.2014.0142

15. Cardoso AA, Reis NM, Vieira MDCS, Borgatto AF, Folle A, Guimarães ACDA. Associated factors and profile of injuries in professional ballroom dancers in Brazil: A crosssectional study. Motriz: J Phys Educ. 2020;26(2):1-8. doi:10.1590/s1980-6574202000020077

16. Eysenbach G. Improving the quality of web surveys: The Checklist for Reporting Results of Internet E-Surveys (CHERRIES). J Med Internet Res. 2004;6(3):1-6.

17. V Vandenbroucke JP, von Elm E, Altman DG, Gøtzsche PC, Mulrow CD, Pocock SJ, et al. Strengthening the Reporting of Observational Studies in Epidemiology (STROBE): explanation and elaboration. Int J Surg. 2014;12(12):150024. doi: 10.1016/j.ijsu.2014.07.014.

18. Bahr R, Clarsen B, Derman W, Dvorak J, Emery CA, Finch $\mathrm{CF}$, et al. International Olympic Committee Consensus Statement: Methods for Recording and Reporting of Epidemiological Data on Injury and Illness in Sports 2020 (Including the STROBE Extension for Sports Injury and Illness Surveillance (STROBE-SIIS)). Orthop J Sports Med.
2020;8(2):2325967120902908. doi: 10.1177/ 2325967120902908.

19. Jacobson BH, Redus B, Palmer T. An assessment of injuries in college cheerleading: distribution, frequency, and associated factors. Br J Sports Med. 2005;39(4):237-40.

20. Hutchinson MR. Cheerleading injuries: Patterns, prevention, case reports. Phys Sportsmed. 1997;25(9):83-96. doi:10.3810/psm.1997.09.1508

21. Gaston RG, Loeffler BJ. Sports-Specific Injuries of the Hand and Wrist. Clin Sports Med. 2015;34(1):1-10.

22. Fridman L, Fraser-Thomas JL, McFaull SR, Macpherson AK. Epidemiology of sports-related injuries in children and youth presenting to Canadian emergency departments from 2007-2010. Sport Med Arthrosc Rehabil Ther Technol. 2013;5(1):1-6. doi:10.1186/2052-1847-5-30

23. Lin CY, Casey E, Herman DC, Katz N, Tenforde AS. Sex Differences in Common Sports Injuries. PM\&R. 2018;10 (10):1073-82. doi:10.1016/j.pmrj.2018.03.008

24. Gornitzky AL, Lott A, Yellin JL, Fabricant PD, Lawrence JT, Ganley TJ. Sport-Specific Yearly Risk and Incidence of Anterior Cruciate Ligament Tears in High School Athletes. Am J Sports Med. 2016;44(10):2716-23. doi:10.1177/ 0363546515617742

25. Bahr R, Holme I. Risk factors for sports injuries - A methodological approach. Br J Sports Med. 2003;37(5):384-92. doi:10.1136/bjsm.37.5.384

26. Meeuwisse WH, Tyreman H, Hagel B, Emery C. A dynamic model of etiology in sport injury: The recursive nature of risk and causation. Clin J Sport Med. 2007;17(3):215-19. doi:10.1097/JSM.0b013e3180592a48

27. Schulz BMR, Marshall SW, Yang J, Mueller FO, Weaver L, Bowling JM. A Prospective Cohort Study of Injury Incidence and Risk Factors in North Carolina High School Competitive Cheerleaders. Am J Sports Med. 2004;32 (2):396-405. doi:10.1177/0363546503261715

\section{Corresponding author}

Isabela Bianchini Marolde. Departamento de Fisioterapia, Universidade Federal de São Carlos, São Carlos, SP, Brasil.

E-mail: isabelabianchini@gmail.com.

Manuscript received on April 24, 2021

Manuscript accepted on May 27, 2021

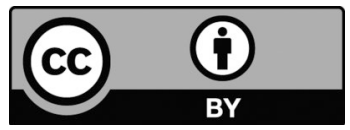

Motriz. The Journal of Physical Education. UNESP. Rio Claro, SP, Brazil - eISSN: 1980-6574 - under a license Creative Commons - Version 4.0 